\title{
Evaluating complete androgen insensitivity syndrome with a multimodal sonography system
}

\author{
Zining Xu ${ }^{1,2} \wedge$, Chengzhong Peng ${ }^{1}$, Liyi Zhang ${ }^{1}$, Qiongxiao Huang ${ }^{3}$ \\ ${ }^{1}$ Department of Ultrasonography, Zhejiang Provincial People's Hospital, People's Hospital of Hangzhou Medical College, Hangzhou, China; \\ ${ }^{2}$ Department of Ultrasonography, Zhejiang Provincial Tongde Hospital, Hangzhou, China; ${ }^{3}$ Department of Reproductive Endocrinology, Zhejiang \\ Provincial People's Hospital, People's Hospital of Hangzhou Medical College, Hangzhou, China
}

Correspondence to: Liyi Zhang, MD. Department of Ultrasonography, Zhejiang Provincial People's Hospital, People's Hospital of Hangzhou Medical College, Hangzhou 310012, China. Email: zhangliyi1982@163.com.

Submitted Sep 06, 2021. Accepted for publication Oct 25, 2021.

doi: 10.21037/qims-21-893

View this article at: https://dx.doi.org/10.21037/qims-21-893

We aim to share our experience in the application of a multimodal sonography system (MMSS) for evaluating complete androgen insensitivity syndrome (CAIS) in a 21-year-old patient with a suspected disorder of sex development (DSD). To the best of our knowledge, no similar studies have been previously reported.

DSD is a group of congenital conditions with mismatch development between the chromosomal, gonadal and anatomical sex. Early diagnosis is essential for selecting an appropriate diagnosis and treatment strategy. The value of sonography lies in the assessment of the development of the internal reproductive tract, gonads, and breasts, and exclusion of upper urinary system malformations and adrenal disorders. In particular, anatomical evaluation of the internal reproductive system is the first and most critical step. Clinically, pelvic sonography is often employed as the first-line imaging tool (1-3). However, this modality is curtailed by its low sensitivity and specificity for evaluating the malformations of the internal reproductive system in DSD $(1,2,4,5)$. In order to improve the diagnostic performance, we combined low-frequency sonography, superficial high-frequency sonography, transvaginal/transrectal sonography, and saline infusion sonosalpingography (SIS) to construct the MMSS for evaluation.

A 21-year-old patient with a female phenotype was admitted to our hospital for primary amenorrhea.
Gynecological examination of the external genitalia showed a female juvenile vulva. The vagina could accommodate the little finger, but the cervix could not be reached. No mass was palpated in the pelvic cavity. The patient's breast size was normal, with relatively small nipples. The pubic and armpit hair was thin. The patient was then referred for a comprehensive sonographic examination, which was performed with the MMSS. The main findings were pooled as follows (Figure 1): (I) a pair of gonads suspected to be testicles, which were smaller than normal testicles, were detected in the upper pelvic cavity; however, ovotestes could not be definitively excluded; (II) under the guidance of sonography, catheterization was performed smoothly, followed by injection of normal saline into the internal reproductive tract. The internal reproductive tract was fully dilated and its dilatability was precisely measured; (III) the internal reproductive tract was inferred to be a blind vagina as it appeared to be a cord-like hypoechoic structure, with no characteristic contour of the uterus, cervix, or endometrium; and there was no fluid accumulation in the pelvic cavity during the process of SIS, which suggesting that the internal reproductive tract did not communicate with the pelvic cavity; (IV) no vaginalurethral confluence, abnormal passages of the urogenital sinus (UGS), or hypospadias were noted; (V) bilateral breasts were well-developed; and (VI) no upper urinary system malformation or abnormalities of the adrenal glands

^ ORCID: 0000-0003-1983-6468. 

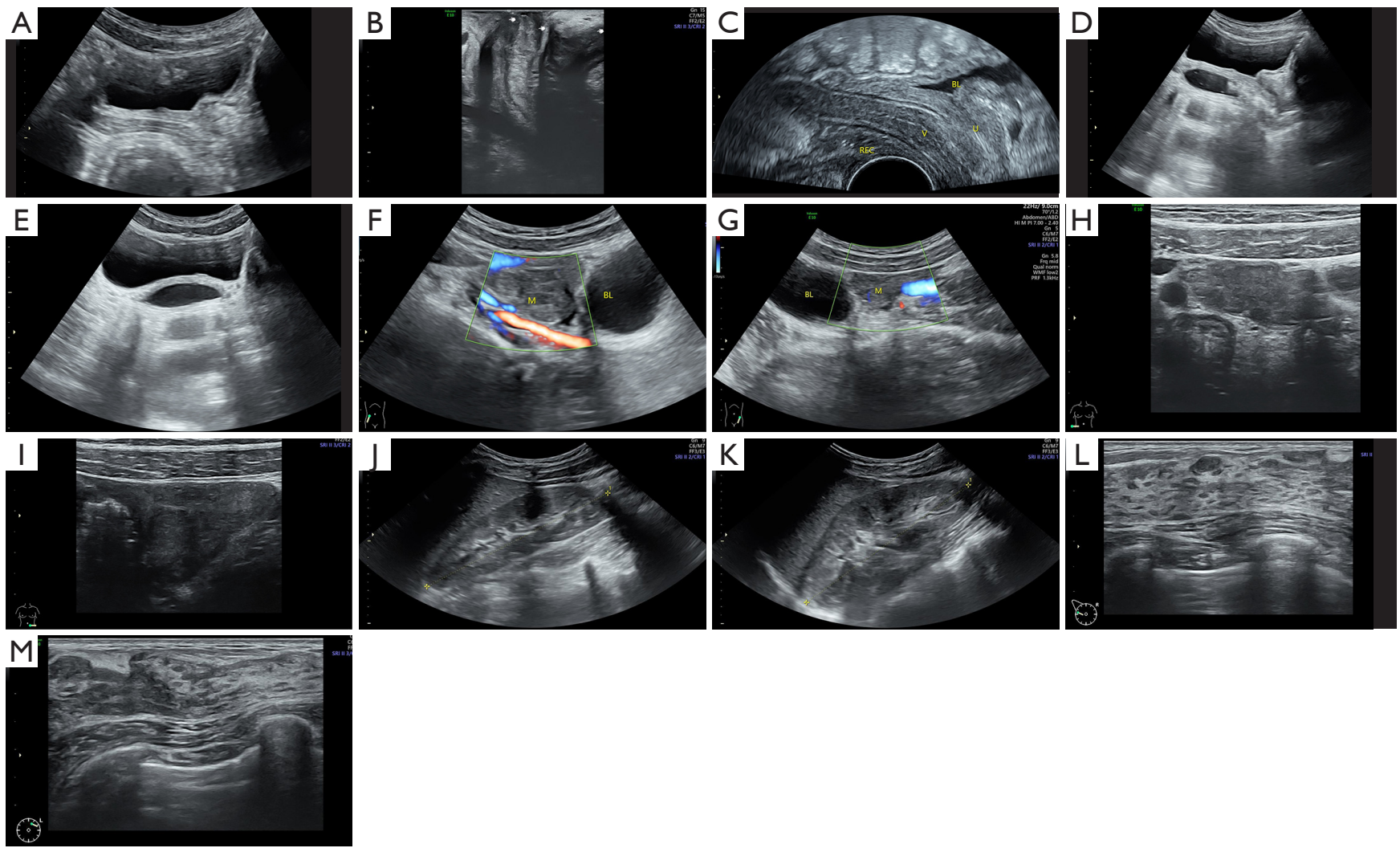

Figure 1 Case evaluation with MMSS. (A-C) The internal reproductive tract was initially examined by transabdominal low-frequency sonography, transperineal superficial high-frequency sonography, and transrectal sonography. The internal reproductive tract appeared to be a cord-like hypoechoic structure, which was located behind the urethra and bladder and in front of the rectum. Its cranial end was approximately $8.0 \mathrm{~cm}$ above the level of the internal urethral orifice, and the tail end communicated with the outside directly. There was no characteristic contour of the uterus, cervix, or endometrium. At the center of the tract, a longitudinal gas line was observed. Neither vaginal-urethral confluence nor corpus cavernosum and cavernous body of the urethra were found. The arrows represent the urethra, the internal reproductive tract, and the rectum, from left to right, respectively. (D,E) SIS was subsequently performed. The vagina was fully dilated, with a length of approximately $14.0 \mathrm{~cm}$, an anteroposterior diameter of approximately $2.0 \mathrm{~cm}$, and a width diameter of approximately $5.0 \mathrm{~cm}$. The inner surface of the vagina was smooth, with no vaginalurethral confluence or other abnormal passages of UGS. During the process of SIS, reflux was observed and there was no fluid accumulation in the pelvic cavity, suggesting that the vagina did not communicate with the pelvic cavity. (Before SIS is conducted, a gynecological examination should be performed by a gynecologist. If there is no common canal of the UGS, preference should be given to internal reproductive tract SIS or internal reproductive tract SIS combined with urethra SIS. In patients who are unsuitable for or refuse to accept internal reproductive tract SIS, urethral SIS alone can be performed. However, if there is a common canal of the UGS, UGS-SIS should be performed. Moreover, if there is an intact hymen, the outside diameter of the catheter must be smaller than the hymen aperture). (F-I) The gonads were observed by transabdominal/transperineal lowfrequency sonography and superficial high-frequency sonography. In the process of searching for the gonads, comprehensive scanning of the pelvic cavity, peri-iliac vessels, hilum, groin and perineum is recommended. We noted a pair of oval gonads in the upper pelvic cavity beside the iliac vessels, with their anterior edges adjacent to the parietal peritoneum. Both gonads, measuring 6.31 and $5.93 \mathrm{~mL}$ on the right and left sides, respectively, were smaller than normal testicles. They were solid, with several hypoechoic nodules inside. Also, no follicle structures or polar differences were noted. Color Doppler flow imaging showed sparse blood flow signal in the parenchyma. A paramesonephric cyst was detected beside each gonad. (J,K) The kidneys appeared to be normal by low-frequency sonography. (L,M) The breasts were well-developed as determined by superficial high-frequency sonography. REC, rectum; V, vaginal; U, urethra; BL, bladder; BL, bladder; M, gonad; MMSS, multimodal sonography system; SIS, saline infusion sonosalpingography; UGS, urogenital sinus. 

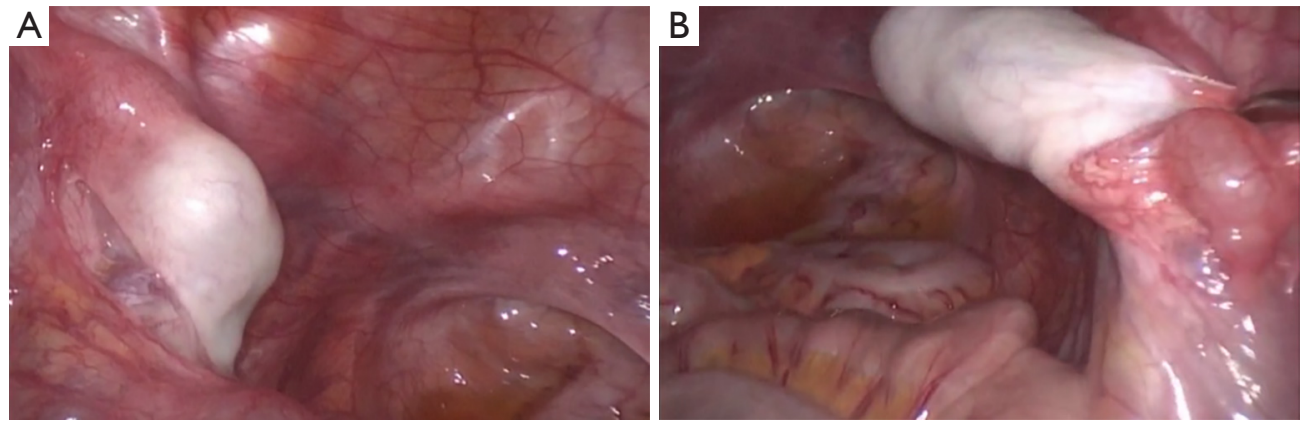

Figure 2 Hysteroscopic-laparoscopic surgery. (A,B) A pair of gonads, both measuring $6.24 \mathrm{~mL}$, were detected in front of the iliac vessels. A paramesonephric cyst around each gonad was noted.
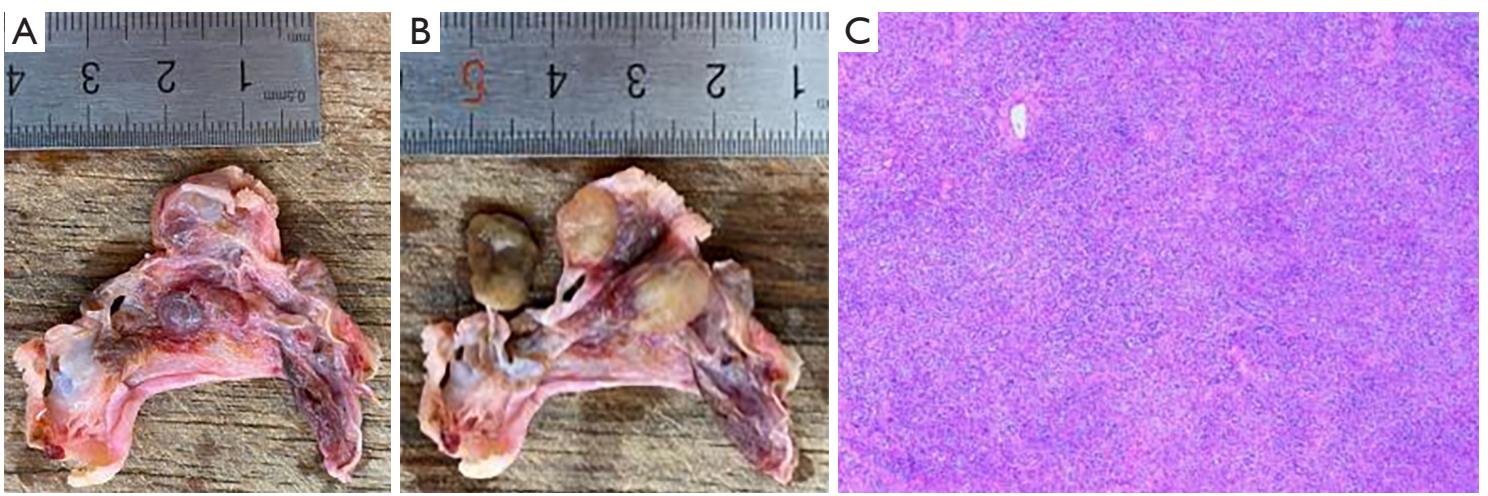

Figure 3 Pathological examination of the resected gonads. (A,B) The gross specimen revealed elliptic, solid gonads, with a pinky-grey, soft, and multiple nodular appearance after sectioning. (C) Microscopic pathology demonstrated the following: (I) nodular hyperplasia, with a fine convoluted seminiferous structure in the bilateral gonads; (II) collagen proliferation; (III) absence of spermatogenic cells; and (IV) obvious proliferation of interstitial cells. All of these features are consistent with undescended testes. Staining method: hematoxylin and eosin staining, magnification: $\times 100$.

were observed. A diagnosis of CAIS was suspected.

Magnetic resonance (MR) and laboratory examinations were subsequently carried out. Based on the MR results, the internal reproductive tract was inferred to be a blind vagina. Also, a pair of gonads beside the iliac vessels were noted. Serum sex hormone examination showed the following: follicle stimulating hormone $8.3 \mathrm{IU} / \mathrm{L}$, luteinizing hormone $26.8 \mathrm{IU} / \mathrm{L}$, estradiol $127 \mathrm{pmol} / \mathrm{L}$, prolactin $99.6 \mathrm{mIU} / \mathrm{L}$, testosterone $17.09 \mathrm{nmol} / \mathrm{L}$, and progesterone $1.43 \mathrm{nmol} / \mathrm{L}$. Karyotyping indicated a genetic male with a karyotype of 46, XY. Whole exome sequencing showed an AR gene mutation: c. $1847 \mathrm{G}>\mathrm{A}$, hemizygous, which has been documented to be pathogenically related to androgen insensitivity syndrome (AIS). Hysteroscopic-laparoscopic surgery was performed within 1 week after sonography. No uterus, fallopian tubes, and ovaries were observed in the pelvic cavity. A blind vagina measuring about $10 \mathrm{~cm}$ long and 2 fingers wide was revealed. A pair of gonads were detected in the upper pelvic cavity (Figure 2) and were resected. Pathological examination showed that both gonads were actually dysplastic testicles (Figure 3). All of these findings further suggested the possibility of CAIS. Estrogen replacement therapy and vaginal dilatation were performed.

CAIS is a rare type of DSD characterized by maternally inherited or de novo mutations involving the androgen receptor. During embryonic development, differentiation of the bilateral Mullerian ducts into the fallopian tubes, uterus, and upper vagina, in which the UGS forms the lower segment of the vagina, is blunted $(6,7)$. Verification of the vagina's presence and determination of its dilatability, especially the length, is an important factor for deciding whether vaginoplasty, an operative treatment, should be given preference or not. By taking advantage of different frequency probes and multiple perspectives, we confirmed 
the existence of a blind vagina and obtained its length when it was fully dilated (approximately $14.0 \mathrm{~cm}$ ). Vaginal dilatation, a non-invasive therapy, was subsequently recommended in this case. Moreover, exclusion of vaginalurethral confluence, hypospadias, and other abnormal passages of the UGS aided in the differentiation of CAIS from other types of DSD, such as partial AIS or 46XX, DSD.

The gonads in this case of CAIS were actually testicles. Androgen produced by the testicles can be converted into estrogen in vivo, so the natural initiation of puberty can still be maintained (6). On the other hand, since undescended testes have a high probability of malignant transformation after puberty, gonadectomy in early adulthood is recommended (8). Therefore, the evaluation of the type and location of the gonads facilitates the determination of the opportunity and scope of surgery. Moreover, evaluation of the type of gonads can distinguish CAIS from Mayer-Rokitansky-Küster-Hauser syndrome, which also often presents with primordial uterus or blind vagina. Nonetheless, the gonads in the former condition are testicles, whereas in the latter, they are ovaries. Lowfrequency sonography and superficial high-frequency sonography play different roles in the assessment of gonads. Low-frequency sonography focuses on the determination of location, while superficial high-frequency sonography has the advantage of observing structural details. Studies have shown that the location of gonads helps to identify their type, as undescended testes are predominantly located at the descending path of normal testicles. In CAIS, 35.7\%, $48.2 \%$ and $16.1 \%$ of gonads are located at the pelvic, groin, and labia majora, respectively (7). By sequentially scanning these regions with low-frequency sonography, we found a pair of gonads in the upper pelvic cavity, which increased the possibility that the gonads were testicles.

Subsequently, detailed structural characterization with superficial high-frequency sonography demonstrated anatomical features that are consistent with undescended testes. The solid nodules in the gonads were consistent with the pathological findings, which might be related to the stagnation of testicular tissue development in a relatively primitive stage. Undescended testes should be differentiated from ovaries and ovotestes, which can be distinguished by location and internal structure (obtained by the MMSS). For women of reproductive age, the ovaries are generally fixed in the adnexal area with follicular structure. The ovotestes, which are composed of both ovarian and testicular tissues in different proportions, are often located at the position of the predominant gonadal type under normal circumstances (9). Ovarian tissue might envelop the testicular tissue in a circular fashion within the ovotestes (10), or the 2 tissues might be located at both poles and connected with each other. Based on the location and structural details, the gonads in this case were suspected to be testicles, but ovotestes could not be completely excluded.

In this report, an MMSS was constructed and applied for the evaluation of a case of CAIS. The integrated information increased the diagnostic confidence for CAIS and provided essential guidance to the physician, which could aid in selecting an appropriate treatment that is most conducive to the patients. It should be noted that it is necessary for clinicians to avoid biased diagnostic guidance. For example, it is inappropriate to make a deterministic judgment on the type of gonads, or suggest that the gonads are absent if no gonad was found by sonography. Further MR examination should still be recommended.

\section{Acknowledgments}

Funding: This study was supported by the Program of Basic Public Welfare Research in Zhejiang Province of China (LGF19H180011) and the Medical and Health Technology Project in Zhejiang Province of China (2020KY430).

\section{Footnote}

Provenance and Peer Review: This article was a standard submission to the journal. The article has undergone external peer review.

Conflicts of Interest: All authors have completed the ICMJE uniform disclosure form (available at https://dx.doi. org/10.21037/qims-21-893). The authors report that this study was supported by the Program of Basic Public Welfare Research in Zhejiang Province of China (No. LGF19H180011), the Medical and Health Technology Project in Zhejiang Province of China (No. 2020KY430), and International Multi-center (Clinical Trial Registration Number: ChiCTR1800015519). The authors have no other conflicts of interest to declare.

Ethical Statement: The authors are accountable for all aspects of the work in ensuring that questions related to the accuracy or integrity of any part of the work are appropriately investigated and resolved. 
Open Access Statement: This is an Open Access article distributed in accordance with the Creative Commons Attribution-NonCommercial-NoDerivs 4.0 International License (CC BY-NC-ND 4.0), which permits the noncommercial replication and distribution of the article with the strict proviso that no changes or edits are made and the original work is properly cited (including links to both the formal publication through the relevant DOI and the license). See: https://creativecommons.org/licenses/by-nc-nd/4.0/.

\section{References}

1. Steven M, O'Toole S, Lam JP, MacKinlay GA, Cascio S. Laparoscopy versus ultrasonography for the evaluation of Mullerian structures in children with complex disorders of sex development. Pediatr Surg Int 2012;28:1161-4.

2. Riccabona M, Darge K, Lobo ML, Ording-Muller LS, Augdal TA, Avni FE, Blickman J, Damasio BM, Ntoulia A, Papadopoulou F, Vivier PH, Willi U. ESPR Uroradiology Taskforce--imaging recommendations in paediatric uroradiology, part VIII: retrograde urethrography, imaging disorder of sexual development and imaging childhood testicular torsion. Pediatr Radiol 2015;45:2023-8.

3. Diamond DA, Yu RN. Disorders of sexual development: etiology, evaluation, and medical management. In: Wein AJ, Kavoussi LR, Novick AC, Partin AW, Peters CA. editors. Campbell-Walsh Urology. 11th edition. Philadelphia, PA, USA: WB Saumders, 2016:3469-97.

Cite this article as: $\mathrm{Xu} \mathrm{Z,} \mathrm{Peng} \mathrm{C,} \mathrm{Zhang} \mathrm{L,} \mathrm{Huang} \mathrm{Q.}$ Evaluating complete androgen insensitivity syndrome with a multimodal sonography system. Quant Imaging Med Surg 2022;12(3):2165-2169. doi: 10.21037/qims-21-893
4. Tasian GE, Copp HL. Diagnostic performance of ultrasound in nonpalpable cryptorchidism: a systematic review and meta-analysis. Pediatrics 2011;127:119-28.

5. Ebert KM, Hewitt GD, Indyk JA, McCracken KA, Nahata L, Jayanthi VR. Normal pelvic ultrasound or MRI does not rule out neoplasm in patients with gonadal dysgenesis and Y chromosome material. J Pediatr Urol 2018;14:154. e1-154.e6.

6. Gulía C, Baldassarra S, Zangari A, Briganti V, Gigli S, Gaffi M, Signore F, Vallone C, Nucciotti R, Costantini FM, Pizzuti A, Bernardo S, Porrello A, Piergentili R. Androgen insensitivity syndrome. Eur Rev Med Pharmacol Sci 2018;22:3873-87.

7. Tian Q, Liu H, Lang J. Clinical characteristics and varieties of complete androgen insensitivity syndrome. Chinese Journal of Practical Gynecology and Obstetrics 2004;20:723-5.

8. Urology Group, Pediatric Surgery Branch, Chinese Medical Association. Chinese expert consensus on disorders of sexual development. Chinese Journal of Pediatric Surgery 2019;40:289-97.

9. Chavhan GB, Parra DA, Oudjhane K, Miller SF, Babyn PS, Pippi Salle FL. Imaging of ambiguous genitalia: classification and diagnostic approach. Radiographics 2008;28:1891-904.

10. Schnitzer JJ, Donahoe PK. Surgical treatment of congenital adrenal hyperplasia. Endocrinol Metab Clin North Am 2001;30:137-54. 